\title{
Development of Water Safety Plan Models for Water Refilling Stations in the Philippines
}

\author{
Victorio B. Molina, ${ }^{1}$ Romeo R. Quizon, ${ }^{1}$ Vivien Fe F. Fadrilan - Camacho, ${ }^{1}$ Catherine Lee-Delos Reyes, ${ }^{1}$ \\ Jovito P. Deauna II, ${ }^{1}$ Hygeia C. Agosto, ${ }^{2}$ Pio Justin V. Asuncion, ${ }^{3}$ Joselito M. Riego de Dios, ${ }^{4}$ \\ Maria Sonabel S. Anarna ${ }^{4}$ and Bonifacio B. Magtibay ${ }^{5}$ \\ ${ }^{1}$ Department of Environmental and Occupational Health, College of Public Health, University of the Philippines Manila \\ ${ }^{2}$ Department of Epidemiology and Biostatistics, College of Public Health, University of the Philippines Manila \\ ${ }^{3}$ Health Research Division, Health Policy Development and Planning Bureau, Department of Health, Philippines \\ ${ }^{4}$ Environment Related Disease Division, Department of Health, Philippines \\ ${ }^{5}$ Environmental Health, World Health Organization, Philippines
}

\begin{abstract}
Objectives. The aim of the project is to develop water safety plan (WSP) guidelines specific for water refilling station (WRS) operators. The guidelines will serve as model for future formulation of WSP among WRS across the country.

Methods. A criteria was developed for selecting WRS. Site visits were initially done along with the selection of eight (8) water refilling stations from pre-identified areas across the country. The operators of the selected WRS together with their respective local sanitary inspectors underwent a workshop where they were trained and guided on the step-by-step process of devising a WSP. Coaching and mentoring was then conducted for the improvement of the draft WSPs. A guideline manual on how to develop WSPs for WRS was drafted to serve as reference for other WRS in the country.
\end{abstract}

Results. The study developed a WSP model specifically made for WRS setting. Eight (8) final drafts of WSP were also accomplished. WRS operators and local sanitary inspectors were trained on how to develop WSP which will support and facilitate appropriate implementation of these functional WSPs.

Conclusion. The formulation of WSPs among WRS ensures delivery of safe drinking- water among its consumers. It serves as an initial step in realizing the vision of $\mathrm{DOH}$ of institutionalizing WSPs among all water utilities in the country. The guidelines developed will be a useful tool for other WRS in the country for preparing their own WSPs.

\footnotetext{
Presented as a poster at the 50th SEAMEO TropMed Philippines Anniversary - Public Health Conference, November 13, 2015, CPH Auditorium, College of Public Health, University of the Philippines Manila.

Corresponding author: Victorio B. Molina, MPH, PhD Department of Environmental and Occupational Health

College of Public Health, University of the Philippines Manila

625 Pedro Gil St., Ermita, Manila 1000 Philippines

Telephone: +6325247102

Email: vbmolina@post.upm.edu.ph
}

Key Words: risk management, water refilling stations, water safety plan

\section{Introduction}

Waterborne diseases which include acute gastroenteritis or diarrhea remain as one of the major health problems globally. Diarrhea accounts for 1.73 million deaths each year ${ }^{1}$ and contributes to 1.7 billion cases of the disease globally. ${ }^{2}$ It is the second leading cause of death in children below 5 years old. ${ }^{2}$ In the Philippines, diarrhea is the $6^{\text {th }}$ leading cause of morbidity in the general population. ${ }^{3}$ It has been noted globally that $88 \%$ of diarrheal cases were attributable to unsafe water supply, sanitation, and hygiene. ${ }^{4}$

Access to safe and quality drinking-water is one of the most important basic needs of human beings and it plays a major role in public health. One of the targets of Millennium Development Goal (MDG) ${ }^{7}$ was to reach half of the proportion of people without access to safe drinkingwater and sanitation worldwide by 2015. ${ }^{5}$ This vision paved the way for the formulation and development of Water Safety Plans (WSP), a framework and guidelines for water service providers to help maintain safe and quality drinking-water. ${ }^{6}$

In other countries, the effectiveness of WSP in minimizing the potential risks and hazards that may compromise the safety of drinking-water is well-recognized. In Nigeria, a study conducted in 2014 found out that only two out of six selected urban areas have properly developed and implemented WSP in their water supply providers. Results showed that absence or poor performance of WSP in the other areas has grave public health consequences since control points of water safety hazards cannot be identified and measures to prevent or eliminate these hazards do not exist. ${ }^{7}$ While in the rural setting, a case study was done in 2015 among two rural water supply utilities in Beijing, China that assessed how WSP can be executed in those particular set-ups. They determined the hazards and risk factors that 
affect water safety, created control measures for improvement of water source protection, operating system management and treatment processes; and established emergency mechanisms. It was then concluded that there is feasibility for WSP to be applied in a rural water supply system. ${ }^{8}$

WSP is a management tool that can be applied in all types of water systems as presented in the abovementioned case studies to ensure the safety and quality of supplied water. It uses a comprehensive risk assessment and management approach from water source (catchment) to consumer. WSPs are comprised of five main components, namely: (a) Health-based targets, (b) System Assessment, (c) Operational Monitoring, (d) Management Plans, and (e) Surveillance. Minimization of contamination of water source, reduction or removal of contamination through treatment processes, and the prevention of contamination during storage, distribution, and handling of drinking water are principles adopted from the multiple- barrier approach as well as Hazard Analysis Critical Control Point (HACCP) strategy used in the food industry. ${ }^{6}$

WSPs were first introduced in developing countries in 2005..$^{9}$ In the Philippines, the strengthening of WSP development and implementation was enforced. Administrative Order 2014-0027 is promulgated to support the full adoption of the WSP model. The AO requires all water service providers to conceptualize and fully implement a WSP specifically designed for their water supply system. ${ }^{10}$ One of these water service providers are the Water Refilling Stations (WRS).

In the Philippines, preference of the public to water from WRS was influenced by previous significant lapses on the part of the public water utilities which resulted to cholera outbreaks linked to contaminated water caused by the illegal connections of water pipes. ${ }^{11}$ As of May 2014, the country has 7,459 water refilling stations registered at the Philippine Business Registry (PBR). The continuous increase in the public's demand for water from WRS emphasizes the need for WRS to have WSPs hence, the need for the conduct of this study.

\section{Materials and Methods}

Literature review and consultations with technical experts on water safety planning and WRS operations were carried out in the development of a water safety plan (WSP) manual tailored fit to the water refilling station (WRS) setting. The manual contained guidelines for WRS operators for the formulation and implementation of their own WSP.

A pilot study was then conducted among eight (8) WRS representing the industry in the country. Study sites were selected based on the type of raw water used by the WRS. Also, pre-determined areas of the country were considered by the project for the selection of participants such as Pangasinan, National Capital Region (NCR), Laguna, Cebu,
Boracay, Cagayan de Oro, and Davao. From the aforementioned sites, the project team selected 2 WRS with private deep well (>60 feet depth), 1 WRS with private shallow well ( $<60$ feet depth), 3 WRS with water district, 1 WRS with LGU-managed water utility, and 1 WRS with bulk/delivered water as raw water. These sites recorded an average of 500 gallons a day production rate depending on their customer's demand. WRS operators were then selected based on the following criteria: (1) operator has undergone Certification Course for Water Refilling Station and Plant Operators (CCWRSPO) conducted by the University of the Philippines Manila - College of Public Health and other Department of Health (DOH) approved institutions; and (2) willingness to participate for the entire duration of the study and its activities. The local sanitary inspectors of the selected areas were also tapped to assist on the selection of WRS and served as coaches of the operators.

A workshop on the development of WSP was conducted among the selected WRS operators together with their respective local sanitary inspectors. The activity provided the participants with the background on the need for the development and implementation of WSP, overview of the WSP process and its content, and an initial draft of WSP specifically designed for their own WRS.

Series of site visits were then conducted to monitor and improve the WSPs of the WRS operators. The project team in collaboration with the local sanitary inspectors initiated a coaching and mentoring activity which aimed to assist the operators on the refinement of the WSP to further enhance the quality of their WSPs. A summary of the approach and methods that were conducted in the study is presented in Figure 1.

\section{Results}

The study formulated a WSP guideline specifically designed for WRS setting. The guideline serves as a model among WRS in the country on devising their own WSPs. The manual provides the step-by-step process needed in formulating a WSP. Moreover, it presents both specifications on the processes involved on the two main product waters of WRS which is purified drinking-water and safe drinkingwater (also known as the mineral water).

Eight (8) WSPs were completed as outputs of the eight operators of water refilling stations trained and assisted by the project. The involvement of the local sanitary inspectors on the activities of the project such as training on the development of WSP and its implementation served as a milestone on being one of the key implementers of environmental health and sanitation in the country.

\section{Discussions}

The conventional approach used to monitor water quality at the tap is found to be ineffective because notification 


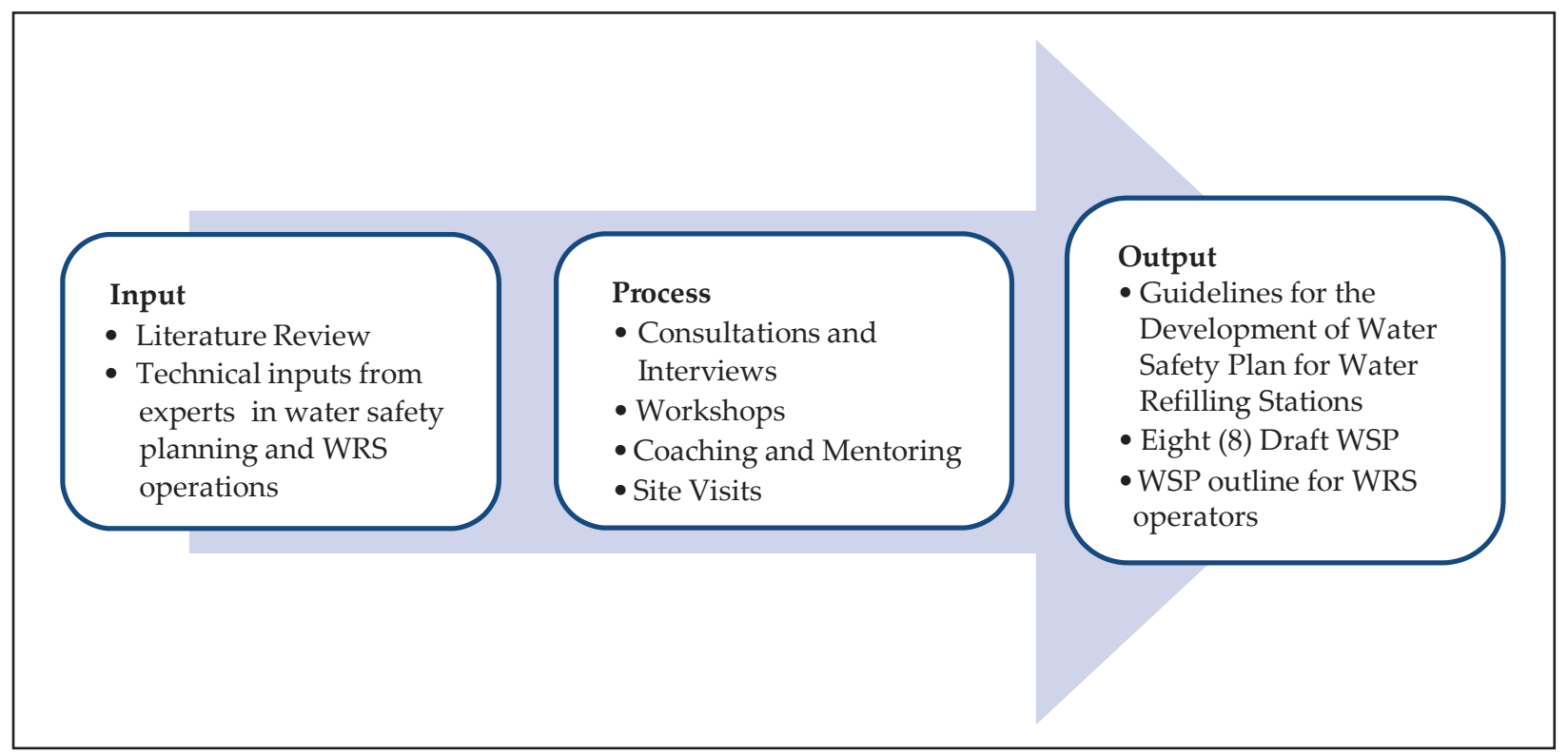

Figure 1. Process flow diagram of the methods conducted in the study.

comes too late whenever there is contamination. Consumers will have already ingested the contaminated water before corrective actions could be made. WSP addresses this gap by ensuring that appropriate control measures are already in place minimizing the risk for contamination. Monitoring is done to prevent any hazardous outcomes. WSPs are conceptualized and implemented to prevent or minimize contamination of water source, remove contamination through treatment, and prevent recontamination during storage, distribution, and handling of drinking-water. ${ }^{12}$

WSP guidelines specifically designed for WRS setting was adopted on the WSP Manual of WHO which consisted of eleven primary steps. ${ }^{6}$ The WSP guidelines for the WRS used the same steps however, modifications were made to tailor fit the phases on the WRS processes. The WSP for the WRS involved the following steps: (1) Designate a Focal Person; (2) Describe the Water Supply System; (3) Identify Hazards and Hazardous Events, and Assess the Risk; (4) Determine and Validate Control Measures, and Prioritize the Risks; (5) Improve/Upgrade Plan; (6) Define Monitoring of the Control Measures; (7) Verify the Effectiveness of the WSP; (8) Develop Management Procedures; (9) Develop Support Programs; (10) Conduct Water Safety Plan Systems Review; and (11) Revise WSP following an incident. ${ }^{13}$

Minor modifications of WHO water safety plan manual were made to address the unique characteristics of WRS. Such unsuitability includes assembling a WSP team composing of a number of experts on the water supply system process. ${ }^{6}$ The modified manual then indicated only the need for a focal person to monitor and implement the WSP which is the WRS owner or operator. ${ }^{13}$
The creation of WSP among WRS warrants delivery of safe drinking-water among the consumers. The development of the WSP guidelines for WRS marked as one of the initial steps of the Department of Health on incorporating the WSP approach in all water service providers in the country. Moreover, the guidelines serve as an important tool to other existing WRS on devising and implementing their own WSPs. Involvement of the local health office particularly the health and sanitation unit is a critical undertaking for the implementation of the WSP. The role of the sanitary inspectors revolves on the monitoring and ensuring compliance among the WRS operators. This serves as an initial step on strict regulation of operational WRS across the country.

On the other hand, the following issues and challenges were encountered by the project team during the conduct of the study: (1) Lack of technical knowledge on the WSP concept both the WRS operators and the concerned LGU implementers; (2) Variation on the involved water system processes among the WRS; and (3) Lack of resources that are deemed necessary to sustain a functional WSP. These identified issues are critical and need to be appropriately addressed in order to attain and sustain functional WSPs for this water system.

\section{Acknowledgments}

The authors acknowledge the funding and technical assistance from the World Health Organization and the Department of Health. Also, the active participation of the WRS operators and local sanitary inspectors is duly appreciated which primarily contributed to the success of this endeavour. 
References

1. Prüss-Üstün A, Kay D, Fewtrell L, Bartram J. Unsafe water, sanitation and hygiene. In: Ezzati M, Lopez AD, Rodgers A, Murray CJL, eds. Comparative Quantification of Health Risks: Global and Regional Burden of Diseases Attributable to Selected Major Risk Factors. Geneva: WHO; 2004.

2. Diarrhoeal disease [Online]. [cited 2015 Oct]. Available from http://www.who.int/mediacentre/factsheets/fs330/en/.

3. Department of Health. Field Health Services Information System: Notifiable Diseases [Online]. 2012 [cited 2015 Oct]. Available from http://nec.doh.gov.ph/images/fhsis2009/fhsis2012.pdf.

4. Centers for Disease Prevention and Control. Global Water, Sanitation, \& Hygiene (WASH) [Online]. 2013 [cited 2015 Oct]. Available from http://www.cdc.gov/healthywater/global/diarrhea-burden.html.

5. United Nations. Fact Sheet on Millennium Development Goal [Online]. 2013 [cited 2015 Oct]. Available from http://www.un.org/millennium goals/pdf/Goal_7_fs.pdf.

6. Bartram J, et al. Water Safety Plan Manual: Step-by-step risk management for drinking-water suppliers [Online]. 2009 [cited 2015 Oct]. Available from http://apps.who.int/iris/bitstream/10665/75141/1/ 9789241562638_eng.pdf.
7. Ezenwaji E, Phil-Eze P. Water Safety Plan as a Tool for Improved Quality of Municipal Drinking Water in Nigeria [Online]. 2014. [cited 2016 Apr]. Available from http://dx.doi.org/10.4236/jep.2014.511100

8. Ye B, Chen Y, Li Y, Li H, Yang L, Wang W. Risk assessment and water safety plan: case study in Beijing, China. J Water Health. 2015; 13(2):510-21.

9. Thompson T, Magtibay B. Recent Developments in Drinking Water Quality Management - Global and Regional Perspectives [Online]. 2006 [cited 2015 Oct]. Available from http://www.jwrcnet.or.jp/aswin/en/ symposium_archive/images/data/007_inv_es_who.pdf.

10. Department of Health. Department of Health Annual Report 2014 [Online]. 2014 [cited 2015 Oct]. Available from http://portal.doh.gov.ph/ sites/default/files/DOH_Annual_Report2014.pdf.

11. Magtibay B. Water refilling station: an alternative source of drinking water supply in the Philippines [Online]. 2004 [cited 2015 Oct]. Available from http://wedc.lboro.ac.uk/resources/conference/30/Magtibay.pdf.

12. World Health Organization. Water Safety Plans: Managing drinkingwater quality from catchment to consumer [Online]. 2005 [cited 2014 May]. Available from http://www.who.int/water_sanitation_health/ dwq/wsp170805.pdf.

13. World Health Organization. Guidelines in Developing Water Safety Plan for Water Refilling Stations in the Philippines. 2015. Unpublished.

\section{Acta Medica Prilippina \\ THE NATIONAL HEALTH SCIENCE JOURNAE \\ पर

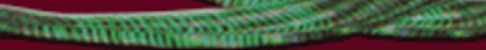

\section{Acta is now accepting membership}

Privileges of members:

- Allows you to submit articles for possible publication

- Have access to all the articles in the website (archives included) which can be downloaded and printed in pdf format

- Advertise your products/services in the available spaces of the website (for approval of the Editor-in-chief)

For details, please visit our website at www. actamedicaphilippina.com.ph or e-mail us at businessmanager@actamedicaphilippina.com.ph for any questions or queries. 